\title{
NeuroSky Mindwave Mobile Headset 2 as an Intervention for Reduction of Stress and Anxiety Measured With Pulse Rate Variability
}

\author{
Habshi Al-Kaf ${ }^{1}$, Ahsan Khandoker ${ }^{1}$, Kinda Khalaf ${ }^{1}$, Herbert F Jelinek ${ }^{1}$ \\ ${ }^{1}$ Khalifa University, Abu Dhabi, United Arab Emirates
}

\begin{abstract}
NeuroSky Mindwave Mobile headset 2 (MMH2) is an EEG-based biofeedback device that can be used to assist in relaxation by providing users feedback on their level of relaxation. In this study, we aimed to assess the effect of using $M M H 2$ on pulse rate variability $(P R V)$ as a measure of relaxation in addition to the in-App real-time data.

Six participants were recruited and provided information about the study once they contacted the university. Participants were required to use the Brainwave Visualizer application as part of the NeuroSky suite for relaxation for 10 minutes. PRV of each participant was determined before and after use of the MMH2. Biosignals were initially preprocessed to remove artifacts and resampled at $8 \mathrm{~Hz}$ for time and frequency domain analysis using purpose written Matlab software. We obtained multiple parameters including the average value of the inter-pulse intervals, standard deviation, root mean square of the successive differences, and stress index,

The stress score from the MMH2 screen indicated a decrease in overall stress by the participants. RMSSD

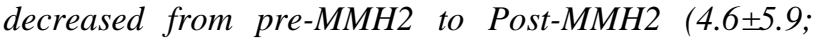
2.9 $\pm 0.9 ; p=)$ whilst the Kubios Stress Index decreased as well $(0.74 \pm 1.5 ; 0.44 \pm 1.1, p=)$

MMH2 can be used to help reduce stress and anxiety levels, making it a potentially useful tool for home use.
\end{abstract}

\section{Introduction}

Portable EEG Apps is a new technology that has great potential in reducing the impact of mental health disorders. EEG recording and analysis has contributed to computer game development, and computer interface design for many years as serious games technologies [1]. However, EEG-based stress reduction technology is difficult to use in certain environments such as classrooms or as home use, which is why portable and easy to use technology has been developed that can utilize EEG recordings not only for in clinic diagnostics but also for home-based stress reduction. For example, Emotiv EPOC is a device that is being used for neurofeedback for attention deficit hyperactivity disorder (ADHD) [2]. Most of the research papers advocate the use of NeuroSky devices (MindWave,
MindWave Mobile, MindBand, and MindSet). On the other hand, Emotiv EPOC was rarely used compared to NeuroSky [3-6]. A comparison of portable EEG technology showed that it was considered a valid instrument for measuring EEG [3].

One of the advantages the MMH2 has is its cost compared to other EEG measuring devices [7]. The MMH2 has the ability to measure attention and relaxation using a dry electrode that is placed on the forehead. The headset records raw EEG data that is sent to a recorder platform through a Bluetooth connection. In addition, the $\mathrm{MMH} 2$ has consistent data recording capacity over long time periods, and data is comparable to that of medical grade devices [7]. However, it has lower accuracy and potential calibration errors and spectral differences at frequencies that are low [7]. Nonetheless, Van (2014) showed that the NeuroSky mindset was able to detect sleep at stage 1 , with $81 \%$ accuracy. It also has the ability to predict and alert a person when they are about to fall asleep (drowsy). For instance, when a person is driving [8]. Furthermore, it was found that it can be used in controlling a wheelchair through data collected by Brain Computer Interfaces (BCI) [9].

In this paper, our aim was to use MMH2 and observe if the relaxation neurofeedback application increases the level of relaxation by analyzing the pre-training and posttraining PRV of the participant.

\section{Methodology}

\subsection{Ethical Compliance}

Participants were informed about the aims and method of the study and provided an informed consent after their agreement to take part in this research.

\subsection{Participants selection and categories}

Six participants were recruited by media advertisement. Participants ages ranged between 60 to 74 years for both males and females. No exclusion or inclusion criteria were set. Participants did not report any mental health disorders or neuropathology that could potentially affect the outcome of the research. 


\subsection{NeuroSky headset application}

PRV measurements were recorded for 10 minutes before and after participants used the MMH2 for 10 minutes. Participants were supported to place the MMH2 sensor on their forehead and clip the other sensors to their left earlobe as shown in Figure 1. Participants were instructed about diaphragmatic breathing as a mindfulness breathing technique to use during their relaxation training [9]. The Brainwave Visualiser application was connected to the $\mathrm{MMH} 2$ by Bluetooth. In order to undergo the relaxation training, participants were required to keep a ball they saw on the screen floating for as much time as possible and as high as possible (Figure 2). The more relaxed the participant was, the higher the ball will float and will spend more time in the air. Participants were able to see their own high scores including "Best Float Time" and "Best Float Height" to follow up with their own progress and see how well they are doing in the relaxation training as part of the neurofeedback. In addition, participants had an infrared sensor attached to their index finger to measure blood volume pulse (BVP) (Thought Technology, Montreal, Canada).

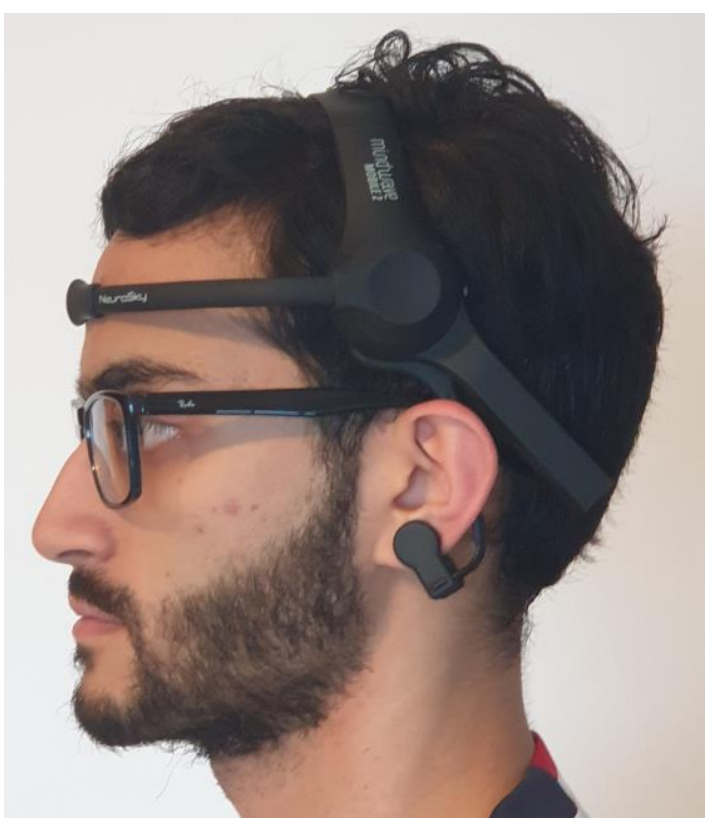

Figure 1. A demonstration of how to wear MMH2.

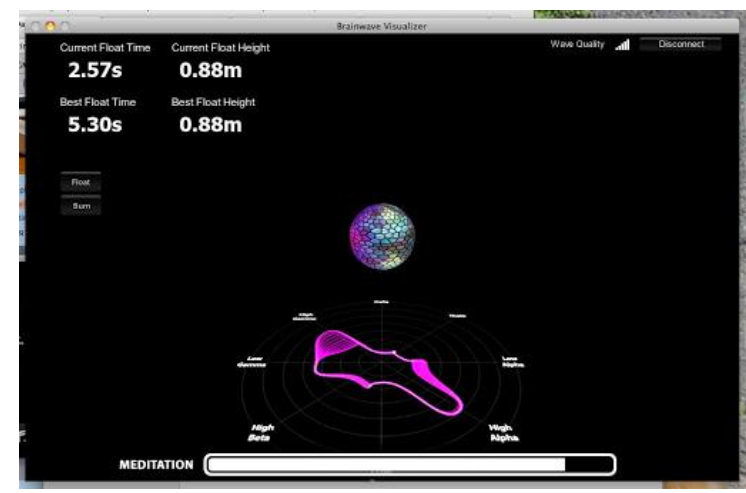

Figure 2. Screenshot of the minigame for the relaxation training. Source: Adapted from [10]

\subsection{Matlab coding for heart rate}

After obtaining the BVP of each participant, a purpose written Matlab code was used to remove ectopic readings and resample it to $8 \mathrm{~Hz}$ in order to obtain the minimum resampling frequency of heart rate, which needs to be evenly sampled for power spectral analysis [11]. Kubios software (Kubios HRV standard 3.3.1, released in August 2019) was used to determine heart rate variability from the $\mathrm{BVP}$ recordings.

\subsection{Analysis of heart rate variability}

The Standard version of the Kubios software [12] was used along with the ProComp5 Infinity System with BioGraph Infinity Software combined with the Physiology Suite (Thought Technology Inc, Canada) to record and analyze the heart rate. The RR intervals for each participant were transferred into Kubios for HRV analysis. From the analysis, we obtained parameters including the mean heart rate intervals (Mean HR), standard deviation (SDNN), root mean square of the successive differences (RMSSD), very low frequency power (VLF), low frequency power (LF), high frequency power (HF), the low frequency to high frequency ratio $(\mathrm{LF} / \mathrm{HF})$, stress index $(\mathrm{SI})$, standard deviation along line-of-identity in the Poincare plot (SD2 $\%$, sympathetic nervous system (SNS) index, and parasympathetic nervous system (PNS) index. Statistical differences were determined using the two tailed t-test and for two dependent means, and significance set at $\mathrm{p}<0.05$. Mann Whitney test was also used for HR data, with significance set at $\mathrm{p}<0.05$

\section{Results}

T value was calculated and found to be 3.9929 , giving us a $\mathrm{p}$ value of 0.0104 . This was found through the difference score, which was used to find t-value. This implies that values are significant at $\mathrm{p}<0.05$.

Mann Whitney test was used to check whether the HR 
recordings of each participant were selected form the same population or not. Table 1 summarizes the obtained $\mathrm{p}$ values.

Table 1. HR recordings pre and post using $\mathrm{MMH} 2$ for each participant

\begin{tabular}{llll}
\hline Participants & Pre HR & Post HR & P-value \\
\hline ButJ & 55.0 & 55.0 & $<0.01$ \\
HabG & 54.0 & 57.0 & $<0.001$ \\
JelH & 46.0 & 48.0 & $<0.001$ \\
PotC & 62.0 & 64.0 & $<0.001$ \\
RicD & 50.0 & 54.0 & $<0.001$ \\
RicP & 56.0 & 58.0 & $<0.001$ \\
\hline
\end{tabular}

Results from table 1 indicate that HR recordings pre- and post-using MMH2 for each participant were selected from the same population. All $\mathrm{p}$ values were $<0.05$, showing that they are all significant at $\mathrm{p}<0.05$

HRV analysis before the use of MMH2 along with the average for each parameter is summarized in Table 2 .

Table 2. Participants' measurements before using $\mathrm{MMH} 2$ for Mean HR, RMSSD, SD2 \%: Standard deviation along line-ofidentity in the Poincare plot, and SI: Stress index.

\begin{tabular}{lllll}
\hline Participants & $\begin{array}{l}\text { Mean } \\
\text { HR }\end{array}$ & RMSSD & $\begin{array}{l}\text { SD2 } \\
\%\end{array}$ & SI \\
\hline ButJ & 55.0 & 2.60 & 87.9 & 11.5 \\
HabG & 54.0 & 16.5 & 95.6 & 3.5 \\
JelH & 46.0 & 4.30 & 83.2 & 12.4 \\
PotC & 62.0 & 2.30 & 89.6 & 25.2 \\
RicD & 50.0 & 0.60 & 92.0 & 25.4 \\
RicP & 56.0 & 1.80 & 90.4 & 25.0 \\
Average & 53.8 & 4.68 & 89.8 & 17.2 \\
\hline
\end{tabular}

HRV analysis after the use of MMH2 along with the average for each parameter is summarized in Table 3.

Table 3. Participants' measurements after using MMH2 for Mean HR, RMSSD, SD2 \%: Standard deviation along line-ofidentity in the Poincare plot, and SI: Stress index. P values from Mann Whitney test were also included in the table.

\begin{tabular}{llllll}
\hline Participants & $\begin{array}{l}\text { Mean } \\
\text { HR }\end{array}$ & RMSSD & $\begin{array}{l}\text { SD2 } \\
\%\end{array}$ & SI & P-value \\
\hline ButJ & 55.0 & 2.20 & 87.6 & 12.1 & 0.0101 \\
HabG & 57.0 & 3.30 & 88.0 & 13.6 & 0.0000 \\
JelH & 48.0 & 2.60 & 84.1 & 13.4 & 0.0000 \\
PotC & 64.0 & 1.80 & 91.0 & 24.4 & 0.0000 \\
RicD & 54.0 & 2.30 & 92.0 & 15.9 & 0.0000 \\
RicP & 58.0 & 4.20 & 93.9 & 6.70 & 0.0000 \\
Average & 56.0 & 2.90 & 89.4 & 14.4 & \\
\hline
\end{tabular}

Figure 3 provides an example of Baevsky's stress index. During stressful situations, SNS increases, resting heart rate is elevated, and HRV is decreased. Sympathetic tone represents the stress of the participant RicD before the use of MMH2.

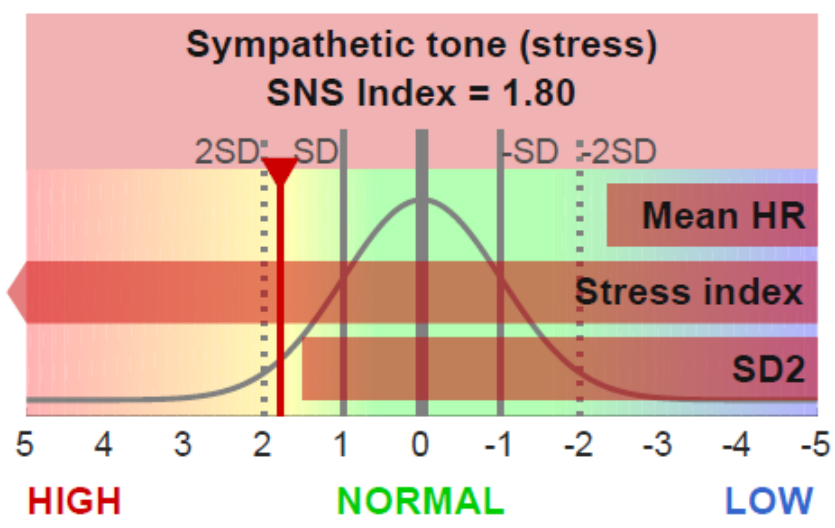

Figure 3. Illustration of the analysis results of Kubios before the use of MMH2. A comparison of the obtained results with normal resting values is shown to observe how the stress score of the participant is affected.

The curve in Figure 3 represents an example of the SNS index before the use of MMH2.

Figure 4 illustrates the analysis results of Kubios after the use of $\mathrm{MMH} 2$. A comparison of the obtained results indicates an improvement in stress score of the participant.

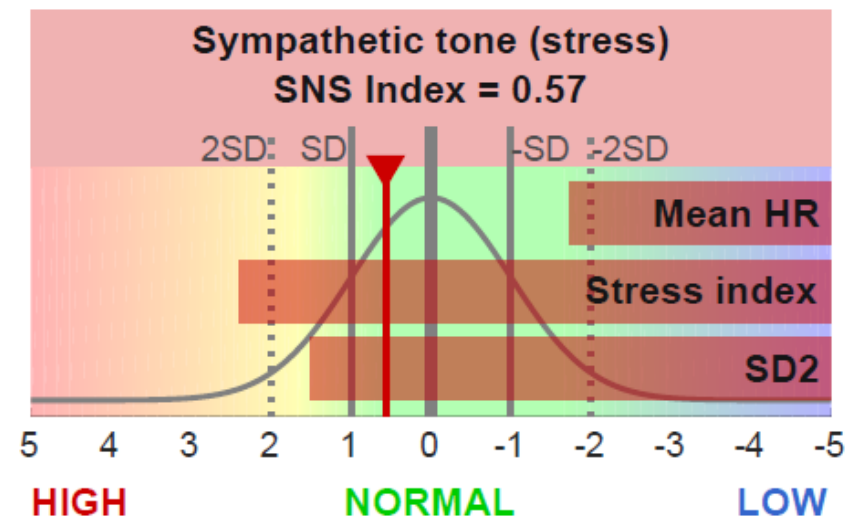

Figure 4. Illustration of the analysis results of Kubios after the use of MMH2. A comparison of the obtained results with normal resting values is shown to observe how the stress score of the participant is affected.

The fact that the average of both RMSSD and stress index decreased from pre-using $\mathrm{MMH} 2$ to post using MMH2 means that the stress score generally decreased for participants; from 4.6 \pm 5.9 to $2.9 \pm 0.9$ in RMSSD and from $0.74 \pm 1.5$ to $0.44 \pm 1.1$ for stress index.

\section{Discussion}

In this study, the use of $\mathrm{MMH} 2$ and its effect on 
relaxation using the associated neurofeedback was assessed. The MMH2 device has been previously used in various studies [13, 14], but not for the evaluation of its stress reduction option.

We believe the main limitation in this study was the number of participants, which made it difficult to extrapolate from the results. However, most of the studies that reported using $\mathrm{MMH} 2$ as an intervention in their experiments consisted of a similar number of participants [15]. As for the decrease observed in the RMSSD, we believe that this might be linked to an increase in stress. However, this might be due to the fact that participants were encouraged to use a resonant frequency breathing that is around 6 breaths per minute $(0.1 \mathrm{~Hz})$. Therefore, this decrease in RMSSD may be due to the shift in the heart rate frequency to $0.1 \mathrm{~Hz}$, which can be considered in the LF (low frequency) domain whereas parasympathetic activity is associated with HF (high frequency) [16].

For future studies, a larger sample is being recruited. Different serious games applications that use $\mathrm{MMH} 2$ can also be used to observe how effective they are for stress reduction. Another aspect that can be added is directly asking each participant after they use the MMH2 about whether they felt any change regarding their stress levels.

\section{Conclusion}

Portable EEG technology can significantly change the way doctors treat mental health disorders, but it needs to be further studied before applying it on a larger scale. Overall, MMH2 showed its abilities in helping reduce stress and anxiety levels, making it possible intervention with a high potential in being a medical tool that can be for home use.

\section{Acknowledgments}

We would like to acknowledge Khalifa University for providing the needed requirements for the study

\section{References}

[1] Hengameh Marzbani, Hamid Reza Marateb, Marjan Mansourian, "Neurofeedback: A Comprehensive Review on System Design, Methodology and Clinical Applications," Basic and Clinical Neuroscience, vol. 7, no. 2, pp. 143-158, 2016.

[2] Q. Wang and O. Sourina, "Real-Time Mental Arithmetic Task Recognition From EEG Signals," in IEEE Transactions on Neural Systems and Rehabilitation Engineering, vol. 21, no. 2, pp. 225-232, March 2013

[3] Chih-Ming Chen, Sheng-Hui Huang, "Web-based Reading Annotation System With an Attention-based Self-regulated Learning Mechanism For Promoting Reading Performance," British Journal of Educational Technology, vol. 45, no. 5, pp. 959-980, 2013.

[4] Chih-Ming Chen, Chung-Hsin Wu "Effects of Different
Video Lecture Types on Sustained Attention, Emotion, Cognitive Load, and Learning Performance," Computers and Education, vol. 80, pp. 108-120, 2015.

[5] I. Ghergulescu, C.H. Muntean, "A Novel Sensor-based Methodology For Learner's Motivation Analysis in Gamebased Learning," Interacting With Computers, vol. 26, no. 4, pp. 305-320, 2014.

[6] J.Xu, B.Zhong, "Review on Portable EEG Technology in Educational Research," Computers in Human Behavior, vol. 81, pp. 340-349, 2018.

[7] Rieiro H, Diaz-Piedra C, Morales JM, Catena A, Romero S, Roca-Gonzalez J, Fuentes LJ, Di Stasi LL, "Validation of Electroencephalographic Recordings Obtained With a Consumer-Grade, Single Dry Electrode, Low-Cost Device: A Comparative Study," Sensors (Basel), vol. 19, no. 2, p. 2808, 2019.

[8] Van Hal B, Rhodes S, Dunne B, Bossemeyer R, "Low-cost EEG-based Sleep Detection.," in 36th Annual International Conference of the IEEE Engineering in Medicine and Biology Society, Chicago, 2014.

[9] K. Permana, S. K. Wijaya, P. Prajitno, "Controlled Wheelchair Based on Brain Computer Interface Using Neurosky Mindwave Mobile 2," in AIP Conference Proceedings, Indonesia, 2019

[10] J. Medeiros, "Relaxation," Meet NeuroSky's Mind Controller Headset, 1 June 2011.

[11] Singh, D., Vinod, K., \& Saxena, S. C. (2004). Sampling Frequency of The RR Interval Time Series For Spectral Analysis of Heart Rate Variability. Journal of Medical Engineering \& Technology, 28(6), 263-272.

[12] "NeuroSky Mindwave 1.0 Download (free trial)," Software.Informer, [Online]. Available: https://neuroskymindwave.software.informer.com/amp/1.0/. [Accessed 18 May 2020].

[13] Tarvainen MP, Niskanen JP, Lipponen JA, Ranta-Aho PO, Karjalainen PA., "Kubios HRV--heart Rate Variability Analysis Software," Computer Methods and Programs in Biomedicine, vol. 113, no. 1, pp. 210-20, 2014.

[14] R. Shadiev, Y.M. Huang, J.P. Hwang, "Investigating The Effectiveness of Speech-to-text Recognition Applications on Learning Performance, Attention, and Meditation", Educational Technology Research and Development, Vol. 65, no. 5, 2017.

[15] R. Shadiev, T.T. Wu, Y.M. Huang, "Enhancing Learning Performance, Attention, and Meditation Using a Speech-totext Recognition Application: Evidence From Multiple Data Sources", Interactive Learning Environments, Vol. 25, no. 2, pp. 249-261, 2017

[16] Fred Shaffer, J.P.Ginsberg, "An Overview of Heart Rate Variability Metrics and Norms," Frontiers in Public Health, vol. 5, p. 258, 2017.

Address for correspondence:

Habshi Al-Kaf., email: 100045890@ku.ac.ae

Department of biomedical engineering, Khalifa University, Zone 1- Abu Dhabi, United Arab Emirates. 